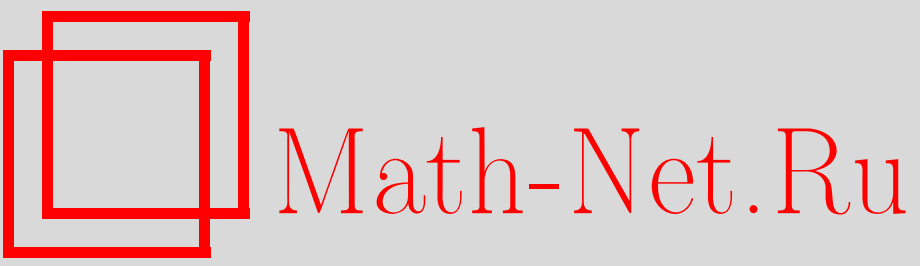

Ф. С. Джепаров, В. Е. Шестопал, Асимптотически точно решаемые модели процессов в стохастически-однородных неупорядоченных решеточных средах, ТМФ, 2003, том 135, номер 1, 117-136

DOI: https://doi.org/10.4213/tmf172

Использование Общероссийского математического портала Math-Net.Ru подразумевает, что вы прочитали и согласны с пользовательским соглашением

http://www.mathnet.ru/rus/agreement

Параметры загрузки:

IP : 54.80 .73 .141

26 апреля 2023 г., 15:12:06 
ТЕОРЕТИЧЕСКАЯ

И МАТЕМАТИЧЕСКАЯ

ФИЗИКА

Том 135, № 1

апрель, 2003

(C) 2003 г. $\quad$ Ф.С. Джепаров* , В.Е. Шестопал*

\section{АСИМПТОТИЧЕСКИ ТОЧНО РЕШАЕМЫЕ МОДЕЛИ ПРОЦЕССОВ В СТОХАСТИЧЕСКИ-ОДНОРОДНЫХ НЕУПОРЯДОЧЕННЫХ РЕШЕТОЧНЫХ СРЕДАХ}

\footnotetext{
Строятся и исследуются асимптотически точно решаемые, в том числе и новые, модели эволюционных процессов в случайных стационарных стохастически-однородных решеточных средах. Впервые дается эффективный метод получения асимптотических разложений при больших временах для пространственных флуктуаций пропагатора во многих изучавшихся ранее, а также новых моделях.
}

Ключевые слова: стохастические процессы, неупорядоченные системы, точно решаемые модели, предельные теоремы.

\section{1.ВВЕДЕНИЕ}

Исследование различных эволюционных процессов в неупорядоченных средах - актуальная область физики и теории случайных процессов [1]-[15]. При решении соответствующих математических задач особый интерес вызывают точно решаемые модели, такие, например, как модель марковских блужданий в случайной среде и различные модели колебаний решетки. Несомненно, важными являются построение и исследование новых разрешимых моделей, имеющих те или иные свойства реальных процессов. В частности, очень интересны асимптотически (в пределе больших времен) точно решаемые (ATP) модели в случайных решеточных средах, соответствующие неоднородно заполненным правильным решеткам со случайным взаимодействием между узлами.

Первая цель предлагаемой работы состоит в построении новых классов точно решаемых стационарных (во времени) стохастически-однородных (в пространстве) случайных систем. Основное внимание уделяется системам, которые не удается представить как малые возмушения каких-либо известных точно решаемых моделей, что свойственно целому ряду активно изучаемых сегодня физических процессов. Мы строим асимптотические разложения (при больших временах) для средних пропагаторов в точно решаемых эволюционных системах, которые очень разнообразны по своей зависимости от локальных случайных параметров среды. Особое внимание мы уделяем алгебраической стороне конструирования новых АТР-моделей. В данной работе отсутствуют

* Институт теоретической и экспериментальной физики, Москва, Россия. E-mail: Victor.Shestopal@itep.ru 
теоремы и полные доказательства, поскольку мы полагаем, что необходимая для обоснования и получения асимптотических разложений техника расчета интегралов и рядов достаточно подробно изложена в работах [10], [14].

Вторая цель данного исследования - изложить метод, позволяющий наряду с асимптотикой среднего пропагатора столь же эффективно оценить асимптотическое поведение его пространственных флуктуаций. Для описываемых в данной работе и предлагавшихся нами прежде моделей эти величины исследовались в гораздо меньшей степени, хотя их важность с различных точек зрения очевидна. В разделе 5 мы указываем необходимые и достаточно простые первые шаги построения асимптотического разложения произвольного среднего произведения матричных элементов пропагатора. После этого остается воспользоваться техникой разложения, развитой в разделах 2 и 3 для пропагатора, и изложенной в статьях [10], [14] методикой обоснования корректности этого разложения. Заметим также, что эта техника разложения высших моментов может служить для исследования разложений пропагаторов и их высших моментов в рассматриваемых нами более широких классах АТР-моделей. Точнее говоря, сначала мы строим и исследуем новые АТР-модели, для которых особую роль играют различные формы обрашения генератора, а затем переходим к еше более широким классам АТР-систем, в расчете которых применяются идеи известного в теории возмушений метода многих параметров, дополненные одновременным применением нескольких преобразований Лапласа.

Точно решаемые модели полезны для оценки различных приближенных и гипотетических подходов, широко используемых в теоретической физике. В связи с этим отметим еше одно важное применение результатов данной работы. Решения, полученные для нового, более обширного класса моделей, дают дополнительный веский аргумент в пользу формального метода "периодической случайной среды”, применяемого в численно-аналитических исследованиях реалистичных процессов блужданий на случайных ("примесных") подмножествах решетки [11]-[13]. Использованная в упомянутых исследованиях методика оказывается совершенно точной для систем, рассматриваемых в настоящей работе. Этим объясняется интерес к описываемому в работе многообразию точно решаемых, хотя и не всегда реалистичных моделей.

Еще одна причина, побуждающая нас привлечь внимание к новым АТР-моделям, состоит в чрезвычайном многообразии новых систем, содержащих в некотором смысле избыточное количество независимых локальных случайных параметров. Некоторые классы таких систем мы приводим. Наличие подобных систем указывает на возможность привести какие-то из этих моделей к реалистическому виду или использовать их в качестве начальных приближений в построениях теории возмушений для физически значимых систем.

1.1. Изучаемые нами эволюционные системы в стохастически-однородных случайных решеточных средах описываются уравнениями вида

$$
\frac{d \widetilde{P}_{x y}^{m n}}{d t}=-\sum_{z} \sum_{l=1}^{N} \tilde{A}_{x z}^{m l} \widetilde{P}_{z y}^{l n},\left.\quad \widetilde{P}_{x y}^{m n}(t)\right|_{t=0}=\delta_{x y} \delta_{m n},
$$

где $x, y, z$ (здесь и ниже) пробегают решетку $\mathbb{Z}^{d}$, индексы $l, m, n=1, \ldots, N$, а параметр $N$ может характеризовать размер элементарной ячейки и/или число компонент 
отнесенной к ячейке векторной физической величины. Мы будем помечать тильдой неусредненные случайные величины. В частности, матричные элементы $\widetilde{A}_{x z}^{m l}$ и $\widetilde{P}_{x z}^{m l}-$ случайные величины. Особое значение для приложений имеет требование стохастической однородности, т.е. инвариантности совместного распределения случайных локальных параметров среды $\tilde{A}_{x z}^{m l}$ по отношению к сдвигам решетки $\tilde{A}_{x z}^{m l} \rightarrow \tilde{A}_{x+a, z+a}^{m l}$ (так называемая "инвариантность в среднем"). Эквивалентное, часто более удобное представление о стохастической пространственной однородности решеточного оператора $F$ связано $c$ возможностью записи этого оператора в виде

$$
F_{x y}^{m n}=\sum_{z} \sum_{l=1}^{N} \alpha_{z x}^{m l} \delta_{x-y, z} \beta_{z y}^{l n}
$$

где для каждого $z$ и всякого $n$ наборы матриц $\left(\alpha_{z x_{1}}, \beta_{z x_{1}} ; \ldots ; \alpha_{z x_{n}}, \beta_{z x_{n}}\right)$ имеют распределение, которое не меняется при любой замене $\left(x_{1}, \ldots, x_{n}\right) \rightarrow\left(x_{1}+a, \ldots, x_{n}+a\right)$. При этом говорят, что случайность носит локальный характер, если соответствующие разным узлам наборы пар матриц $\left\{\left(\alpha_{z x_{1}}, \beta_{z x_{1}}\right) \mid z \in \mathbb{Z}^{d}\right\}$ и $\left\{\left(\alpha_{z x_{2}}, \beta_{z x_{2}}\right) \mid z \in \mathbb{Z}^{d}\right\}$ независимы, когда $x_{1}$ и $x_{2}$ достаточно далеки друг от друга, либо если эта зависимость характеризуется корреляционными функциями, которые достаточно быстро убывают с увеличением расстояния $\left|x_{1}-x_{2}\right|$. Локальный характер случайности среды (т.е. генератора) очень важен для построения и обоснования асимптотик в предлагаемых нами методах. Впрочем, во многих случаях для получения асимптотического разложения достаточно локальности лишь по некоторым пространственным координатам, например когда случайные параметры зависят лишь от части компонент индексируюших их векторов и указанная выше независимость относится именно к этим компонентам.

Стохастическая инвариантность имеет место в реалистичных задачах физики решеточных систем, ее учет важен также и для изучения возможных свойств процессов в некристаллических средах, хорошо описываемых в терминах непрерывного предела неупорядоченных решеточных систем. Основные подразумеваемые здесь процессы - случайные необрываюшиеся цепи Маркова, характерные для блужданий. Они описываются (прямыми или обратными) уравнениями Колмогорова. В этом случае генератор имеет спектр в правой полуплоскости и нулевую точку в непрерывном спектре.

Далее, говоря о топологии в пространстве вектор-функций, мы будем иметь в виду либо топологию гильбертова пространства со скалярным произведением

$$
(f, g)=\sum_{x \in \mathbb{Z}^{d}} \sum_{n=1}^{N} \overline{f_{x}^{n}} g_{x}^{n}
$$

либо $l_{1}$-топологию нормы

$$
\|f\|=\sum_{x \in \mathbb{Z}^{d}} \sum_{n=1}^{N}\left|f_{x}^{n}\right| .
$$




\section{2. ФОРМАЛЬНО-АНАЛИТИЧЕСКОЕ СТРОЕНИЕ НОВЫХ МОДЕЛЕЙ}

Перейдем к методам построения новых в данной проблематике классов АТР-систем. Мы надеемся, что получаемые нами классы моделей настолько обширны и богаты произвольными параметрами, что могут явиться основой для корректных расчетов реалистических систем при помощи подходящих методов теории возмущений. Предлагаемый нами метод построения точно решаемых моделей процессов в случайных решеточных средах пригоден прежде всего для систем, генераторы которых эффективно обратимы (возможно, после их специального бесконечно малого возмушения). Для более явного описания таких операторов и действий над ними мы, начиная с относительно простых генераторов, будем строить новые с помощью умножения, размещения на диагоналях блочно-треугольных операторов и других приемов.

Мы будем активно использовать резольвенты генераторов, т.е. лаплас-образы пропагаторов. Эти объекты очень удобны для формально-алгебраических выкладок. Кроме того, во многих важных для приложений случаях известна связь между поведением резольвенты при малых значениях спектрального параметра и поведением пропагатора при больших временах (см., например, [10], [16]). Здесь прежде всего имеется в виду применение абелевых теорем, которые, с одной стороны, дают точное соответствие между степенными, степенно-логарифмическими и им подобными членами разложений при малых значениях параметров преобразования Лапласа и, с другой стороны, членами асимптотических разложений больших времен.

2.1. Рассматривавшиеся ранее классы точно решаемых моделей марковских случайных блужданий в стохастически-однородных неупорядоченных средах относились к одному из следуюших типов, которые характеризуются свойствами генератора $\tilde{A}$ в пространстве конечномерных вектор-функций на решетке $\mathbb{Z}^{d}$.

А. В работах $[10],[14]$ изучались модели с генераторами вида

$$
\tilde{A}=\alpha B \beta,
$$

где $B$ - трансляционно-инвариантный (т.е. $B_{x, y}^{m n}=B_{x-y, 0}^{m n}$ ) неслучайный оператор, а $\alpha$, $\beta$ - случайные операторы с диагональными по решеточным переменным матричными элементами вида $\alpha_{x y}^{m n}=\alpha_{x}^{m n} \delta_{x y}, \beta_{x y}^{m n}=\beta_{x}^{m n} \delta_{x y}$ с одинаково распределенными при разных $x$ значениями пар $(N \times N)$-матриц $\left(\alpha_{x}^{m n}, \beta_{x}^{m n}\right)$. Этот класс моделей включает многие из первых нетривиальных АТР-систем случайных блужданий в случайной среде с неограниченным в пространстве переносом [10], что впоследствии удалось совместить с требованием симметричности (или пространственной обратимости) интенсивностей переходов при сушественно многомерном движении [14].

Для построения асимптотик в этих системах могут применяться разложения [14]

$$
\begin{aligned}
\frac{1}{\lambda+\alpha B \beta} & =\beta^{-1} \frac{1}{\lambda(\beta \alpha)^{-1}+B} \alpha^{-1}= \\
& =\frac{1}{\lambda}\left(1-\alpha B \frac{1}{\lambda(\beta \alpha)^{-1}+B} \alpha^{-1}\right)=\frac{1}{\lambda}\left(1-\beta^{-1} \frac{1}{\lambda(\beta \alpha)^{-1}+B} B \beta\right), \\
& \frac{1}{\lambda(\beta \alpha)^{-1}+B}=\sum_{s=0}^{S}(G \lambda \eta)^{s} G+(G \lambda \eta)^{S+1} \frac{1}{\lambda(\beta \alpha)^{-1}+B}
\end{aligned}
$$


где

$$
\eta=\left\langle(\beta \alpha)^{-1}\right\rangle-(\beta \alpha)^{-1}, \quad G=\frac{1}{\left\langle\lambda(\beta \alpha)^{-1}\right\rangle+B} .
$$

Использование различных разложений позволило, в частности, наряду с требованием сушествования конечных моментов у достаточного числа величин $\left(\beta_{x} \alpha_{x}\right)^{-r}, r=$ $1,2,3, \ldots$, предполагать сушествование средних не у всех величин $\alpha_{x}, \beta_{x},\left(\alpha_{x}\right)^{-1}$, $\left(\beta_{x}\right)^{-1}, \alpha_{x}^{+} \alpha_{x}, \beta_{x} \beta_{x}^{+}$одновременно, а лишь у некоторых их сочетаний.

Б. В работе [14] отмечалась возможность естественного обобщения указанного выше класса за счет допушения недиагональных по решеточным переменным операторов $\alpha$ и $\beta$, но таких, что сами эти операторы и их обратные имеют представление в виде конечных сумм

$$
\alpha_{x y}^{m n}=\sum_{j=1}^{J} \alpha_{x}^{j ; m n} \delta_{x, y+v_{j}}
$$

В. В литературе рассматривались примеры моделей с генераторами вида

$$
\tilde{A}=B \alpha C
$$

где имеются два неслучайных пространственно-однородных оператора $B$ и $C$, а $\alpha$-оператор, описанный в п. "А” (см., например, [1], [4]). Для получения асимптотики систему (6) можно свести к типу (3) с помощью тождества

$$
\frac{1}{\lambda+B \alpha C}=\frac{1}{\lambda}\left(1-B \alpha \frac{1}{\lambda+C B \alpha} C\right) .
$$

Это же преобразование применимо и при выборе $\alpha$ согласно п. "Б".

2.2. Перейдем к представлению основных алгебраических особенностей предлагаемых в данной работе конструкций АТР-моделей. Мы будем широко использовать возможность обрашения разнообразных операторов. Рассмотрим различные классы эффективно обратимых (или почти обратимых) операторов. Будем называть почти обратимыми операторы, которые обратимы на всюду плотном подпространстве и становятся всюду эффективно обратимыми ограниченными операторами при подходяшем их бесконечно малом возмушении. Другими словами, оператор $C$ почти обратим, если для достаточно малых положительных $\varepsilon$ эффективно обратим оператор $C(\varepsilon)$, причем $\lim _{\varepsilon \rightarrow+0} C(\varepsilon)=C$.

Ниже предлагаются основные конструкции, используемые для получения новых генераторов из имеющихся. Мы берем в качестве начальных операторы (3) и (6).

1. Первый способ конструирования точно решаемых моделей из уже известных состоит в увеличении размерности $N$ в каждом узле решетки за счет "обрашения" метода окаймления в кольце почти обратимых операторов, что выглядит особенно просто в случае образования блочно-треугольных генераторов. В этом частном случае мы полагаем новый генератор равным конечноблочной операторной матрице вида

$$
\tilde{A}=\left(\begin{array}{cccc}
\tilde{A}_{11} & 0 & 0 & \ldots \\
\tilde{A}_{21} & \tilde{A}_{22} & 0 & \ldots \\
\ldots & \ldots & \ldots & \ldots
\end{array}\right)
$$


где диагональные блоки $\tilde{A}_{i i}$ являются почти обратимыми операторами - генераторами АТР-процессов (с меньшими размерностями в каждом узле). В качестве лежаших ниже диагонали блоков $\tilde{A}_{l \neq m}$ можно брать стохастически-однородные операторы весьма обшей структуры.

В общем случае $(M \times M)$-блочного матричного генератора $\tilde{A}$ с эффективно обратимыми диагональными блоками поступаем следующим образом. Сначала определяем цепочку матриц убывающего размера, формально полагая

$$
A_{i j}^{(1)}=A_{i j}, \quad A_{i j}^{(n+1)}=A_{i j}^{(n)}-A_{i n}^{(n)} A_{n n}^{(n)^{-1}} A_{n j}^{(n)}, \quad i, j=n+1, \ldots, N .
$$

Такая цепочка естественно возникает при построении обратного к $\tilde{A}$ оператора методом окаймления, т.е. путем решения системы линейных уравнений над операторными модулями с помощью последовательного исключения неизвестных. Затем выбираем в качестве $A_{n n}^{(n)}, n=1, \ldots, M$, произвольные эффективно обратимые операторы. Далее берем в качестве недиагональных блоков конструируемого генератора достаточно произвольные стохастически-однородные операторы, после чего с помошью вьшеуказанных рекуррентных соотношений легко находим диагональные блоки нужного генератора. Такая процедура дает, в частности, генераторы вида

$$
\begin{gathered}
\left(\begin{array}{ccc}
A_{11}^{(1)} & A_{12} \\
A_{21} & A_{21} A_{11}^{(1)^{-1}} & A_{12}+A_{22}^{(2)}
\end{array}\right) \\
\left(\begin{array}{ccc}
A_{11}^{(1)} & A_{12} & A_{13} \\
A_{21} & A_{21} A_{11}^{(1)}{ }^{-1} A_{12}+A_{22}^{(2)} & A_{23} \\
A_{31} & A_{32} & A_{32}^{(2)} A_{22}^{(2)}{ }^{-1} A_{23}^{(2)}+A_{31} A_{11}^{(1)} A_{13}+A_{33}^{(3)}
\end{array}\right),
\end{gathered}
$$

где $A_{32}^{(2)}=A_{32}-A_{31} A_{11}^{(1)^{-1}} A_{12}$ и $A_{23}^{(2)}=A_{23}-A_{23} A_{11}^{(1)}{ }^{-1} A_{13}$.

Можно взять в качестве $A_{n n}^{(n)}$ лишь почти обратимые операторы, однако в этом случае выбор недиагональных блоков конструируемого генератора ограничен требованием возможности корректного доопределения вспомогательной цепочки. В случае треугольной матрицы никаких проблем нет. В более общем случае заменим операторы вида $A_{n n}^{(n)}$ их возмушениями (вспомним об их почти обратимости) и потребуем, чтобы недиагональные элементы имели подходящие возмущения, обеспечивающие конечность членов цепочки в нулевом по возмущению пределе.

Эта процедура имеет широкие и сушественные обобшения, основанные на иных возможностях обрашения блочных операторов. Так, например, мы можем исключать неизвестные на основе не отдельных уравнений (т.е. строк матришы), а их групп. Кроме того, исключив несколько неизвестных, мы можем получить пространственно-однородный блочный оператор, явно обратимый методом преобразования Фурье. Мы также можем использовать более общую блочно-матричную структуру генератора, соответствующую паре разложений $V_{1} \oplus V_{2} \oplus \cdots=W_{1} \oplus W_{2} \oplus \cdots$ пространства вектор-функций, что позволяет строить генератор из блоков вида $\tilde{A}_{i j}: V_{i} \rightarrow W_{j}$ (в рассмотренной выше конструкции разложения по $V$ и $W$ совпадали).

2. Многие генераторы, строяшиеся с помошью процедуры следуюшего вида, также доступны для исследования нашими методами. 
Пусть блочные операторы $V, W$ взаимно обратны, т.е.

$$
\sum_{l} V^{m l} W^{l n}=\delta_{m n}=\sum_{l} W^{m l} V^{l n},
$$

и эффективно обратим оператор $1+\sum_{n l} H^{n} W^{n l} G^{l}$. В этом случае новый генератор имеет вид

$$
\widetilde{C}^{m n}=G^{m} H^{n}+V^{m n},
$$

а его формальный обратный -

$$
W^{m n}-\left(\sum_{l} W^{m l} G^{l}\right) \frac{1}{1+\sum_{n l} H^{n} W^{n l} G^{l}}\left(\sum_{l} H^{l} W^{l n}\right) .
$$

Предложенный здесь метод также можно обобщать и уточнять.

3. Имея несколько почти обратимых операторов (действуюших в одном пространстве), строим новые генераторы как их произведение

$$
\widetilde{B}=\widetilde{B}^{1} \widetilde{B}^{2} \ldots \widetilde{B}^{M}
$$

4. Тензорные произведения почти обратимых генераторов также, очевидно, почти обратимы. Отметим, что тензорные произведения одинаковых операторов имеют хорошо известные инвариантные подпространства, состоящие из симметричных, антисимметричных и других типов тензоров, что позволяет рассматривать ограничения произведений на указанные подпространства. Важным моментом здесь является "уменьшение локальности" локальных случайных параметров, которые становятся независимыми от части координат решеточного индекса, т.е. становятся функциями на фактор-решетке.

5. Очень мощные средства предоставляют различные обобщения конструкции сплетения операторов. В простейшей форме они основаны на том, что из равенства $B_{1} X=$ $X B_{2}$ следует равенство $\left(\lambda+B_{1}\right)^{-1} X=X\left(\lambda+B_{2}\right)^{-1}$. Так, для $B_{1}=(F H)^{m}$ и $B_{2}=$ $(H F)^{m}$ ( $m$-натуральное число), положив $X=F$, приходим к выражению резольвенты генератора $(F H)^{m}$ вида $($ ср. $(7))$

$$
\begin{aligned}
{\left[\lambda+(F H)^{m}\right]^{-1} } & =\lambda^{-1}\left\{1-(F H)^{m}\left[\lambda+(F H)^{m}\right]^{-1}\right\}= \\
& =\lambda^{-1}\left\{1-F(H F)^{m-k-1}\left[\lambda+(H F)^{m}\right]^{-1}(H F)^{k} H\right\} .
\end{aligned}
$$

Приведем более обшие примеры. Пусть операторы $\left\{A, V_{n}, W_{n}, H_{n}\right\}$ удовлетворяют равенствам $A V_{n}=V_{n} W_{n}$ и $\sum_{n} V_{n} H_{n}=1$. Тогда имеет место соотношение

$$
\frac{1}{\lambda+A}=\sum_{n} V_{n} \frac{1}{\lambda+W_{n}} H_{n}
$$

Действительно, равенство $(\lambda+A) V_{n}=V_{n}\left(\lambda+W_{n}\right)$ влечет равенства $(\lambda+A)^{-1} V_{n}=$ $V_{n}\left(\lambda+W_{n}\right)^{-1}$ и $\sum_{n}(\lambda+A)^{-1} V_{n} H_{n}=\sum_{n} V_{n}\left(\lambda+W_{n}\right)^{-1} H_{n}$. Отметим, что здесь мы имеем также и другой взгляд на использование блочной структуры оператора.

6. Как уже говорилось, в данной работе предлагается метод исследования асимптотики пространственных флуктуаций пропагаторов. Дополнительные средства, которые 
будут для этого использованы, позволяют также рассчитывать пропагаторы, получаемые в нижеследующих примерах.

6а. Система с генератором, являющимся суммой попарно перестановочных генераторов АТР-моделей.

6б. Пусть операторы $\left\{A, F_{n}, G_{n}, H_{n}, I_{n}, J_{n}, K_{n}\right\}$ удовлетворяют соотношениям $H_{n} I_{n}=K_{n} H_{n}, G_{n} K_{n}=K_{n} G_{n}, A F_{n}=F_{n}\left(G_{n}+K_{n}\right)$. Тогда величина

$$
P(t)=\sum_{n} F_{n} e^{-t G_{n}} H_{n} e^{-t I_{n}} J_{n}
$$

удовлетворяет уравнению

$$
\frac{d P(t)}{d t}=-A P(t),\left.\quad P(t)\right|_{t=0}=\sum_{n} F_{n} H_{n} J_{n}
$$

Действительно,

$$
\begin{aligned}
\frac{d P(t)}{d t} & =-\sum_{n}\left[F_{n} G_{n} e^{-t G_{n}} H_{n} e^{-t I_{n}} J_{n}+F_{n} e^{-t G_{n}} H_{n} I_{n} e^{-t I_{n}} J_{n}\right]= \\
& =-\sum_{n} F_{n}\left[G_{n}+K_{n}\right] e^{-t G_{n}} H_{n} e^{-t I_{n}} J_{n}=-A \sum_{n} F_{n} e^{-t G_{n}} H_{n} e^{-t I_{n}} J_{n} .
\end{aligned}
$$

6в. Пусть операторы $\left\{A, F_{n}, G_{n}, H_{n}, I_{n}, J_{n}, K_{n}\right\}$ удовлетворяют соотношениям $G_{n} H_{n}+H_{n} I_{n}=K_{n} H_{n}, G_{n} K_{n}=K_{n} G_{n}, A F_{n}=F_{n} K_{n}$. Тогда величина (10) удовлетворяет уравнению (11). В этом случае, дифференцируя, получим

$$
\begin{aligned}
\frac{d P(t)}{d t} & =-\sum_{n}\left[F_{n} e^{-t G_{n}} G_{n} H_{n} e^{-t I_{n}} J_{n}+F_{n} e^{-t G_{n}} H_{n} I_{n} e^{-t I_{n}} J_{n}\right]= \\
& =-\sum_{n} F_{n} e^{-t G_{n}} K_{n} H_{n} e^{-t I_{n}} J_{n}=-\sum_{n} F_{n} K_{n} e^{-t G_{n}} H_{n} e^{-t I_{n}} J_{n}= \\
& =-A \sum_{n} F_{n} e^{-t G_{n}} H_{n} e^{-t I_{n}} J_{n} .
\end{aligned}
$$

6г. Пусть операторы $\left\{A, C, F_{n}, G_{n}, H_{n}, I_{n}, J_{n}\right\}$ удовлетворяют соотношениям $A F_{n}=$ $F_{n} G_{n}, I_{n} J_{n}=J_{n} C$. Тогда величина (10) удовлетворяет уравнению

$$
\frac{d P(t)}{d t}=-A P(t)-P(t) C,\left.\quad P(t)\right|_{t=0}=\sum_{n} F_{n} H_{n} J_{n} .
$$

Действительно,

$$
\frac{d P(t)}{d t}=-\sum_{n}\left[F_{n} G_{n} e^{-t G_{n}} H_{n} e^{-t I_{n}} J_{n}+F_{n} e^{-t G_{n}} H_{n} e^{-t I_{n}} I_{n} J_{n}\right]
$$

Для последних рассмотренных случаев на основе решений для АТР-моделей с генераторами $G_{n}$ и $J_{n}$ и при довольно обших стохастически-однородных операторах $C, F_{n}$, $H_{n}, I_{n}, K_{n}$ будет описан метод построения асимптотики. 


\section{3. АНАЛИТИЧЕСКИЕ ТРЕБОВАНИЯ К ГЕНЕРАТОРАМ НОВЫХ МОДЕЛЕЙ}

Важной составной частью вычисления и обоснования разложения пропагаторов в предлагаемых моделях является обобшение развитой нами техники разложения резольвенты по флуктуациям обратного генератора, которые при этом автоматически оказываются умноженными на спектральный параметр [10], [14]. Здесь рассматривается общая схема получения разложений. Начнем с рассмотрения АТР-систем, имеющих почти обратимые генераторы, получающиеся, в частности, без применения процедур, описанных в п. 6 предыдушего раздела.

Известна тесная связь между асимптотикой пропагатора на больших временах и асимптотикой при малых по абсолютной величине значениях параметра лаплас-образа пропагатора (или резольвенты генератора полугруппы). Рассмотрим соответствуюшие разложения резольвент построенных выше генераторов $\tilde{A}$.

3.1. Начнем с краткого рассмотрения более простого случая - разложения резольвенты по флуктуациям обратных степеней генератора. Используем соотношение

$$
\frac{1}{\lambda+\tilde{A}}=\frac{1}{\lambda}\left(1-\frac{1}{\lambda \tilde{A}^{-1}+1}\right) .
$$

Дальнейшее разложение ведется по флуктуациям величины $\lambda / \tilde{A}$,

$$
\begin{aligned}
\frac{1}{\lambda \tilde{A}^{-1}+1} & =\frac{1}{\left\langle\lambda \tilde{A}^{-1}\right\rangle+1-\left(\left\langle\lambda \tilde{A}^{-1}\right\rangle-\lambda \tilde{A}^{-1}\right)}= \\
& =\sum_{n=0}^{N} G\left[\left(\left\langle\frac{\lambda}{\tilde{A}}\right\rangle-\frac{\lambda}{\tilde{A}}\right) G\right]^{n}+\widetilde{R}_{N+1}, \quad G=\frac{1}{\left\langle\lambda \tilde{A}^{-1}\right\rangle+1} .
\end{aligned}
$$

Остаточному члену удобно придать одну из следуюших форм:

$$
\widetilde{R}_{N+1}=\left[G\left(\left\langle\frac{\lambda}{\tilde{A}}\right\rangle-\frac{\lambda}{\tilde{A}}\right)\right]^{N+1} \frac{1}{\lambda \tilde{A}^{-1}+1}=\frac{1}{\lambda \tilde{A}^{-1}+1}\left[G\left(\left\langle\frac{\lambda}{\tilde{A}}\right\rangle-\frac{\lambda}{\tilde{A}}\right)\right]^{N+1} .
$$

Это разложение применимо (при соответствующих аналитических допущениях) к системам (3) при $\alpha=1$ или $\beta=1$ и к системам (6). С его помошью, в частности, воспроизводятся многие результаты из работ [10], [14]. Однако для наиболее интересных систем вида (3) необходимо некоторое видоизменение, к изложению которого мы переходим.

3.2. Предположим, что $\tilde{A}=\widetilde{B} \widetilde{C}$ и имеет место достаточно хорошая обратимость оператора $\widetilde{C} \widetilde{B}$. Рассмотрим видоизмененную схему разложения резольвенты случайного процесса вблизи малых значений спектрального параметра по флуктуациям обратного генератора,

$$
\begin{aligned}
\frac{1}{\lambda+\widetilde{A}} & =\frac{1}{\lambda+\widetilde{B} \widetilde{C}}=\frac{1}{\lambda}\left(1-\widetilde{B} \widetilde{C} \frac{1}{\lambda+\widetilde{B} \widetilde{C}}\right)=\frac{1}{\lambda}\left(1-\widetilde{B} \frac{1}{\lambda+\widetilde{C} \widetilde{C}} \widetilde{C}\right)= \\
& =\frac{1}{\lambda}\left[1-\widetilde{B}\left(\frac{1}{\widetilde{C} \widetilde{B}}\right) \frac{1}{\lambda(\widetilde{C} \widetilde{B})^{-1}+1} \widetilde{C}\right]=\frac{1}{\lambda}\left[1-\widetilde{B} \frac{1}{\lambda(\widetilde{C} \widetilde{B})^{-1}+1}\left(\frac{1}{\widetilde{C} \widetilde{B}}\right) \widetilde{C}\right]
\end{aligned}
$$


Дальнейшее разложение ведется по флуктуациям величины $\lambda /(\widetilde{C} \widetilde{B})$,

$$
\begin{aligned}
\frac{1}{\lambda(\widetilde{C} \widetilde{B})^{-1}+1} & =\frac{1}{\left\langle\lambda(\widetilde{C} \widetilde{B})^{-1}\right\rangle+1-\left(\left\langle\lambda(\widetilde{C} \widetilde{B})^{-1}\right\rangle-\lambda(\widetilde{C} \widetilde{B})^{-1}\right)}= \\
& =\sum_{n=0}^{N} G(\lambda \widetilde{\Psi} G)^{n}+\widetilde{R}_{N+1},
\end{aligned}
$$

где

$$
\begin{gathered}
G=\frac{1}{\left\langle\lambda(\widetilde{C} \widetilde{B})^{-1}\right\rangle+1}, \quad \widetilde{\Psi}=\left\langle\frac{1}{\widetilde{C} \widetilde{B}}\right\rangle-\frac{1}{\widetilde{C} \widetilde{B}}, \\
\widetilde{R}_{N+1}=(\lambda G \widetilde{\Psi})^{N+1} \frac{1}{\lambda(\widetilde{C} \widetilde{B})^{-1}+1}=\frac{1}{\lambda(\widetilde{C} \widetilde{B})^{-1}+1}(\lambda \widetilde{\Psi} G)^{N+1} .
\end{gathered}
$$

Учтем также возможное присутствие "обкладок" резольвенты, связанное с рассмотрением систем 5 п. 2.2 , и будем далее рассматривать разложения величин вида

$$
V \frac{1}{\lambda+\tilde{A}} W=V \frac{1}{\lambda+\widetilde{B} \widetilde{C}} W=\cdots
$$

3.3. Выбор вида разложения в соотношениях (14), а также вида остаточного члена $\widetilde{R}_{N+1}(16)$ важен, поскольку позволяет рассматривать разные допушения относительно генератора. В дальнейшем ради определенности мы ограничимся случаем выбора в (14) и в формуле для $\widetilde{R}_{N+1}(16)$ выражений, стоящих перед последними знаками равенства, соответственно.

Сформулируем основные предположения о генераторе, почти всегда явно указывая лишь решеточные индексы.

УСловиЕ 1. Величины $B, C, V$ и $W$ имеют вид

$$
\begin{aligned}
B_{x_{0} x_{r+1}} & =\sum_{s=1}^{S} \sum_{x_{1}, \ldots, x_{r}}\left(B_{s}^{1}\right)_{x_{0}-x_{1}} \xi_{s x_{1}}^{1}\left(B_{s}^{2}\right)_{x_{1}-x_{2}} \ldots \xi_{s x_{r}}^{r}\left(B_{s}^{r+1}\right)_{x_{r}-x_{r+1}} \\
C_{x_{0} x_{r+1}}= & \sum_{s=1}^{S} \sum_{x_{1}, \ldots, x_{r}}\left(C_{s}^{1}\right)_{x_{0}-x_{1}} \xi_{s x_{1}}^{1}\left(C_{s}^{2}\right)_{x_{1}-x_{2}} \ldots \xi_{s x_{r}}^{r}\left(C_{s}^{r+1}\right)_{x_{r}-x_{r+1}} \\
V_{x_{0} x_{r+1}}= & \sum_{s=1}^{S} \sum_{x_{1}, \ldots, x_{r}}\left(V_{s}^{1}\right)_{x_{0}-x_{1}} \xi_{s x_{1}}^{1}\left(V_{s}^{2}\right)_{x_{1}-x_{2}} \ldots \xi_{s x_{r}}^{r}\left(V_{s}^{r+1}\right)_{x_{r}-x_{r+1}} \\
W_{x_{0} x_{r+1}} & =\sum_{s=1}^{S} \sum_{x_{1}, \ldots, x_{r}}\left(W_{s}^{1}\right)_{x_{0}-x_{1}} \xi_{s x_{1}}^{1}\left(W_{s}^{2}\right)_{x_{1}-x_{2}} \ldots \xi_{s x_{r}}^{r}\left(W_{s}^{r+1}\right)_{x_{r}-x_{r+1}}
\end{aligned}
$$

и в подобном виде представляется величина

$$
\left((\widetilde{C} \widetilde{B})^{-1}\right)_{x_{0} x_{r+1}}=\sum_{s=1}^{S} \sum_{x_{1}, \ldots, x_{r}}\left(F_{s}^{1}\right)_{x_{0}-x_{1}} \xi_{s x_{1}}^{1}\left(F_{s}^{2}\right)_{x_{1}-x_{2}} \ldots \xi_{s x_{r}}^{r}\left(F_{s}^{r+1}\right)_{x_{r}-x_{r+1}},
$$


операторы $F_{s}^{1}, \ldots, F_{s}^{r+1}$ представимы в виде сумм

$$
F_{s x y}^{j}=\sum_{a} f_{s}^{j}(a) \delta_{x, y+a}, \quad j=2, \ldots, r, \quad s=1, \ldots, S,
$$

причем для $F_{s}^{2}, \ldots, F_{s}^{r}$ суммы должны быть конечными. Здесь случайными являются величины $\xi_{s x_{j}}^{j}$ - матричные функции на решетке, элементы которых представляют собой рациональные функции локальных случайных параметров среды, таких как матричные элементы $\alpha_{x}^{m n}, \beta_{x}^{m n}$ в (4), $\alpha_{x}^{j ; m n}$ в (5) и, возможно, другие. Заметим, что для систем типа (3) без перехода от $\alpha A \beta$ к $A \beta \alpha$ или $\beta \alpha A$ эти условия в сколько-нибудь интересных случаях не выполняются.

Перепишем величины $\widetilde{\Psi}_{x_{1} x_{r+1}}$ из $(16)$ в виде

$$
\sum_{s=1}^{S} \sum_{x_{1}, \ldots, x_{r}}\left(F_{s}^{1}\right)_{x_{0}-x_{1}} \ldots\left(F_{s}^{r+1}\right)_{x_{r}-x_{r+1}}\left(\left\langle\xi_{s x_{1}}^{1} \ldots \xi_{s x_{r}}^{r}\right\rangle-\xi_{s x_{1}}^{1} \ldots \xi_{s x_{r}}^{r}\right)
$$

УСловиЕ 2 . Набор величин $\left(\xi_{s x_{v}}^{v} \mid v=1, \ldots, r, s=1, \ldots, S\right)$ имеет инвариантное к сдвигам $x_{v} \rightarrow x_{v}+a$ распределение. Два упорядоченных набора величин $\left(\xi_{s x_{v}}^{v} \mid v=\right.$ $1, \ldots, r, s=1, \ldots, S)$ и $\left(\xi_{s y_{v}}^{v} \mid v=1, \ldots, r, s=1, \ldots, S\right)$ должны быть независимы, если минимальное расстояние между входящими в них $x_{v}$ и $y_{v}$ больше заданной конечной величины $R_{\text {corr }}^{(1)}$. Это требование будет выполнено, если в исходных генераторах и вспомогательных операторах случайные параметры имеют конечный радиус коррелящии, а тензорные произведения в построениях не использовались. Как учитывать тензорные произведения, будет показано ниже.

Введем обозначение $\eta_{s x_{1} \ldots x_{r}}=\left\langle\xi_{s x_{1}}^{1} \ldots \xi_{s x_{r}}^{r}\right\rangle-\xi_{s x_{1}}^{1} \ldots \xi_{x_{s r}}^{r}$ и перепишем матричный элемент $\left[G(\lambda \widetilde{\Psi} G)^{n}\right]_{x_{0}^{1} x_{r+1}^{n}} n$-го члена суммы $(15)$ в виде

$$
\begin{aligned}
& \lambda^{n} \sum_{s_{1}, \ldots, s_{n}} \sum_{x_{1}^{1}, \ldots, x_{r+1}^{1}, x_{1}^{2}, \ldots, x_{r}^{n}} G_{x_{0}^{1}-x_{1}^{1}} \times \\
& \quad \times \prod_{j=1}^{n}\left[\left(F_{s_{j}}^{1}\right)_{x_{1}^{j}-x_{2}^{j}} \ldots\left(F_{s_{j}}^{r}\right)_{x_{r}^{j}-x_{r+1}^{j}} \eta_{s_{j} x_{2}^{j} \ldots x_{r+1}^{j}} G_{x_{r+1}^{j}-x_{1}^{j+1}}\right] .
\end{aligned}
$$

Здесь, как и выше, явно указаны лишш решеточные индексы, а произведение некоммутирующих величин определяется правилом $\prod_{j=1}^{n+1} X_{j}=\left(\prod_{j=1}^{n} X_{j}\right) X_{j+1}$.

Поскольку величины $G$ и $F$ не случайны, а среднее значение каждого $\eta_{s x_{1} \ldots x_{r}}$ равно нулю, вклад в усредненное выражение (18) вносят лиш те слагаемые, в которых для каждого сомножителя вида $\eta_{s_{1} x_{1} \ldots x_{r+1}}$ найдется еще один сомножитель $\eta_{s_{2} y_{1} \ldots y_{r+1}}$ с достаточно близким набором решеточных индексов. Это приводит к сокрашению области суммирования, оставляя "конечную окрестность" подрешетки значительно меньшей "размерности”.

В этих предположениях обобщаются требования, при которых результирующий порядок малости по $\lambda$ вблизи нуля оказывается не меньше некоторой линейно растушей вместе с $n$ степени параметра Лапласа. С этой целью налагается еще один набор условий. 
УСловиЕ 3. Операторы $B_{s}^{1}, \ldots, B_{s}^{r}, C_{s}^{2}, \ldots, C_{s}^{r+1}, V_{s}^{1}, \ldots, V_{s}^{r+1}, W_{s}^{1}, \ldots, W_{s}^{r+1}$ ограничены;

- всевозможные операторы $B_{s_{1}}^{r+1} G(\lambda) F_{s_{2}}^{1}, B_{s_{1}}^{r+1}\left\langle(\widetilde{C} \widetilde{B})^{-1}\right\rangle G(\lambda) F_{s_{2}}^{1}, F_{s_{1}}^{r+1} G(\lambda) C_{s_{2}}^{1}$ и $F_{s_{1}}^{1} G(\lambda) F_{s_{2}}^{r+1}$ определены при $\operatorname{Re} \lambda \leqslant 0$ (за возможным исключением $\lambda=0$ ) и убывают как $O\left(|\lambda|^{-1}\right)$ при $|\lambda| \rightarrow \infty$;

- при $\lambda \rightarrow 0, \operatorname{Re} \lambda>0$, нормы операторов $B_{s_{1}}^{r+1}\left\langle(\widetilde{C} \widetilde{B})^{-1}\right\rangle G(\lambda) F_{s_{2}}^{1}, B_{s_{1}}^{r+1} G(\lambda) F_{s_{2}}^{1}$ и $F_{s_{1}}^{r+1} G(\lambda) C_{s_{2}}^{1}$ растут не быстрее некоторой степени $|\lambda|$;

- для всевозможных наборов $T_{a_{1}}, T_{a_{2}}, \ldots$ операторов сдвига решеточного аргумента на векторы $a_{1}, a_{2}, \ldots$ длины не большей, чем $R_{\text {corr }}^{(1)}+r R_{\text {corr }}$, нормы произведений операторов $\prod_{j=1}^{n}\left(T_{a_{j}} F_{s_{j}}^{1} G(\lambda) F_{s_{j}^{\prime}}^{r+1}\right)$ при $\lambda \rightarrow 0, \operatorname{Re} \lambda>0$, растут не быстрее $C|\lambda|^{-v n-\phi}$, где $v<1$

- величины $\left|\left\langle\left(\prod_{j=1}^{r} \alpha_{s_{j}^{1}}^{j}\right)\left(\prod_{j=1}^{n} \eta_{s_{j}^{2} x_{2}^{j} \ldots x_{r+1}^{j}}\right)\left(\prod_{j=1}^{r} \beta_{s_{j}^{3}}^{j}\right)\right\rangle\right|$ с увеличением $n$ растут не быстрее некоторой, не зависящей от наборов $s_{j}^{1}, s_{j}^{2}, s_{j}^{3}$ геометрической прогрессии.

При выполнении всех перечисленных условий усредненные члены разложения резольвенты генератора при $\lambda \rightarrow 0, \operatorname{Re} \lambda>0$, имеют с ростом $n$ необходимое асимптотическое убывание порядка $O\left(\left|\lambda^{n(1-v)}\right|\right)$. Такое асимптотическое поведение станет понятным, если заметить, что вместе с существенным сокращением области суммирования происходит сближение решеточных индексов матричных элементов различных сомножителей вида $F_{s_{1}}^{1} G(\lambda) F_{s_{2}}^{r+1}$. Сами эти сомножители присутствуют в большем количестве, чем все остальные, и нормы их произведений растут вблизи $\lambda=0$ медленнее, чем $|\lambda|^{-1}$.

Оценку асимптотического убывания остаточного члена можно получить при тех же условиях и почти таким же образом, если предварительно воспользоваться неравенством Коши-Шварца,

$$
\begin{aligned}
& \left|\left\langle\left\{V \widetilde{B}\left(\frac{1}{\widetilde{C} \widetilde{B}}\right)(\lambda G \widetilde{\Psi})^{N+1} \frac{1}{\lambda(\widetilde{C} \widetilde{B})^{-1}+1} \widetilde{C} W\right\}_{x y}^{m n}\right\rangle\right|^{2} \leqslant \\
& \leqslant \sum_{z, l}\left\langle\left\{\frac{1}{\lambda(\widetilde{C} \widetilde{B})^{-1}+1} \widetilde{C} W\right\}_{z y}^{l n}\left\{\frac{1}{\lambda(\widetilde{C} \widetilde{B})^{-1}+1} \widetilde{C} W\right\}_{z y}^{l n}\right\rangle \times \\
& \quad \times \sum_{z, l}\left\langle\left\{V \widetilde{B}\left(\frac{1}{\widetilde{C} \widetilde{B}}\right)(\lambda G \widetilde{\Psi})^{N+1}\right\}_{x z}^{m l} \frac{\left\{V \widetilde{B}\left(\frac{1}{\widetilde{C} \widetilde{B}}\right)(\lambda G \widetilde{\Psi})^{N+1}\right\}_{x z}^{m l}}{\{}\right\rangle .
\end{aligned}
$$

Это соотношение подсказывает нам последнее недостаюшее требование к генератору.

УСловиЕ 4. Величина

$$
\sum_{z, l}\left\langle\left\{\left(\lambda(\widetilde{C} \widetilde{B})^{-1}+1\right)^{-1} \widetilde{C} W\right\}_{z y}^{l m} \overline{\left\{\left(\lambda(\widetilde{C} \widetilde{B})^{-1}+1\right)^{-1} \widetilde{C} W\right\}_{z y}^{l m}}\right\rangle
$$

определена при $\operatorname{Re} \lambda \geqslant 0$ (за возможным исключением $\lambda=0$ ) и растет не быстрее некоторой степени $O(|\lambda|)$ при $|\lambda| \rightarrow \infty$; при $\lambda \rightarrow 0, \operatorname{Re} \lambda>0$, ее норма также растет не быстрее некоторой степени $|\lambda|$.

Отметим, что условия 1-4 во многих интересных случаях проверяются достаточно просто (см., например, [10]). 
3.4. В случае нелокальных случайных параметров среды, получающихся из систем с локальными параметрами в результате использования тензорных произведений, сокращение числа членов в решеточных суммах происходит с меншей, однако достаточной для асимптотической сходимости скоростью. Этот случай следует рассматривать почти так же, как и вышеописанный, представляя решеточный индекс в виде набора более коротких целочисленных векторов, “помнящих" тензорные произведения, которые применялись при построении генератора. Это позволяет контролировать существенные и несущественные координаты (переменные) локальных случайных параметров и их влияние на замедление сходимости.

3.5. В случае более сложных генераторов вопрос о возможности совместного обращения случайных операторов на достаточно богатом общем подпространстве может представлять значительные трудности. Поэтому более предпочтительной с аналитической точки зрения, т.е. значительно более удобной для доказательств, представляется нижеследующее видоизменение разложения (15). Для его изложения кратко рассмотрим вопросы регуляризации, а также сохранения почти обратимости при нашем конструировании генераторов АТР-систем.

Начнем с того, что в сумме и остаточном члене $(15)$ величины $\lambda /(\widetilde{C} \widetilde{B})$ и $\langle\lambda /(\widetilde{C} \widetilde{B})\rangle$ всегда оказываются умноженными на $G=(\langle\lambda /(\widetilde{C} \widetilde{B})\rangle+1)^{-1}$. С учетом особенностей наших конструкций генераторов не сложно вводить сингулярности таким образом, что в окончательных выражениях они будут “сокрашаться".

Переходя к более детальному объяснению, отметим, что почти обратимые генераторы могут быть записаны в виде $\widetilde{B}=\widetilde{B}(\hat{\varepsilon})+\Delta(\hat{\varepsilon})$ с обратимым оператором $\widetilde{B}(\hat{\varepsilon})$ и исчезаюшей при $\hat{\varepsilon} \rightarrow 0$ добавкой $\Delta(\hat{\varepsilon})$. В случае перемножения генераторов сохранение почти обратимости также очевидно. Следует положить

$$
\widetilde{B}^{1} \widetilde{B}^{2} \ldots \widetilde{B}^{M}=\left(\widetilde{B}^{1}+\tilde{\varepsilon}^{1}\right)\left(\widetilde{B}^{2}+\tilde{\varepsilon}^{2}\right) \ldots\left(\widetilde{B}^{M}+\tilde{\varepsilon}^{M}\right)+\Delta(\hat{\varepsilon})=\widetilde{B}(\hat{\varepsilon})+\Delta(\hat{\varepsilon})
$$

где исчезающие при $\lambda \rightarrow+0$ ненулевые операторы $\tilde{\varepsilon}^{m}$ подбираются таким образом, чтобы превратить “неполную" обратимость каждого сомножителя во вполне корректную и столь же эффективную обратимость его возмущения. Сомножители, имеющие ограниченные обратные, не нуждаются в указанном возмушении.

Для исходных генераторов вида (4) возможна регуляризация, которая достигается следующими заменами:

$$
\alpha A \beta=\alpha\left(A+\frac{\tilde{\varepsilon}_{0}}{1+\operatorname{Sp}\left(\alpha \alpha^{+}\right)+\operatorname{Sp}\left(\beta \beta^{+}\right)}\right) \beta
$$

где величины $\tilde{\varepsilon}_{0}$ малы при малых значениях $\lambda$ и обеспечивают эффективную обратимость каж дого из сомножителей вида $\left(A^{j}+\tilde{\varepsilon}_{j}\right)$. Отметим, что при такой регуляризации получаются асимптотики в системах с генератором (4) при более обших предположениях, чем это было сделано ранее в работах [10], [14].

С учетом вышесказанного вновь строим разложение резольвенты по флуктуациям обратного к “возмушенному генератору" $\widetilde{C}(\hat{\varepsilon}) \widetilde{B}(\hat{\varepsilon})$, рассматривая сумму малых величин 
$\lambda$ и возмущения как один малый параметр (ср. с формулой (14)),

$$
\begin{aligned}
\frac{1}{\lambda+\widetilde{B} \widetilde{C}}= & \frac{1}{\lambda}\left(1-\widetilde{B} \frac{1}{\lambda+\widetilde{C} \widetilde{B}} \widetilde{C}\right)=\frac{1}{\lambda}\left(1-\widetilde{B} \frac{1}{\lambda+\Delta(\hat{\varepsilon})+\widetilde{C}(\hat{\varepsilon}) \widetilde{B}(\hat{\varepsilon})} \widetilde{C}\right)= \\
= & \frac{1}{\lambda}\left[1-\widetilde{B}\left(\frac{1}{\widetilde{C}(\hat{\varepsilon}) \widetilde{B}(\hat{\varepsilon})}\right) \frac{1}{(\lambda+\Delta(\hat{\varepsilon}))(\widetilde{C}(\hat{\varepsilon}) \widetilde{B}(\hat{\varepsilon}))^{-1}+1} \widetilde{C}\right]= \\
& =\frac{1}{\lambda}\left[1-\widetilde{B} \frac{1}{(\lambda+\Delta(\hat{\varepsilon}))(\widetilde{C}(\hat{\varepsilon}) \widetilde{B}(\hat{\varepsilon}))^{-1}+1}\left(\frac{1}{\widetilde{C}(\hat{\varepsilon}) \widetilde{B}(\hat{\varepsilon})}\right) \widetilde{C}\right] .
\end{aligned}
$$

Дальнейшее разложение ведется по флуктуациям величины $(\lambda+\Delta(\hat{\varepsilon})) /(\widetilde{C}(\hat{\varepsilon}) \widetilde{B}(\hat{\varepsilon}))$,

$$
\begin{aligned}
& \frac{1}{(\lambda+\Delta(\hat{\varepsilon}))(\widetilde{C}(\hat{\varepsilon}) \widetilde{B}(\hat{\varepsilon}))^{-1}+1}= \\
& \quad=\frac{1}{\left\langle(\lambda+\Delta(\hat{\varepsilon}))(\widetilde{C}(\hat{\varepsilon}) \widetilde{B}(\hat{\varepsilon}))^{-1}\right\rangle+1-\widetilde{\Omega}}=\sum_{n=0}^{N} G \widetilde{\Omega}^{n}+\widetilde{R}_{N+1},
\end{aligned}
$$

где

$$
\begin{gathered}
G=\frac{1}{\left\langle(\lambda+\Delta(\hat{\varepsilon}))(\widetilde{C}(\hat{\varepsilon}) \widetilde{B}(\hat{\varepsilon}))^{-1}\right\rangle+1}, \quad \widetilde{\Omega}=\left\langle\frac{\lambda+\Delta(\hat{\varepsilon})}{\widetilde{C}(\hat{\varepsilon}) \widetilde{B}(\hat{\varepsilon})}\right\rangle-\frac{\lambda+\Delta(\hat{\varepsilon})}{\widetilde{C}(\hat{\varepsilon}) \widetilde{B}(\hat{\varepsilon})}, \\
\widetilde{R}_{N+1}=(G \widetilde{\Omega})^{N+1} \frac{1}{(\lambda+\Delta(\hat{\varepsilon}))(\widetilde{C}(\hat{\varepsilon}) \widetilde{B}(\hat{\varepsilon}))^{-1}+1}= \\
=\frac{1}{(\lambda+\Delta(\hat{\varepsilon}))(\widetilde{C}(\hat{\varepsilon}) \widetilde{B}(\hat{\varepsilon}))^{-1}+1}(\widetilde{\Omega} G)^{N+1} .
\end{gathered}
$$

Последующие действия с усредненными членами сумм в (20) основаны на локальном характере случайности параметров в сомножителях генератора. Это приводит если и не к полному исчезновению большинства членов в усредняемых решеточных суммах произведений флуктуаций (и понижению размерностей решеток, по которым ведется суммирование), то к исчезновению соответствуюших произведений с точностью до произвольно задаваемых нами регуляризуюших возмушений (и в этом некоторое, хотя и несушественное, усложнение). Их малость должна выбираться пренебрежимой по сравнению с интересующим нас в окончательном разложении участком асимптотической шкалы. Так, для получения степенной асимптотики пропагатора при малых значениях $\lambda$ величины $\varepsilon_{j}$ в соотношении (19) можно выбрать порядка $e^{-|\lambda|^{-1}}$. Все это позволяет несколько упростить проверку приведенных в п. 3.3 условий 1-4.

3.6. Отметим, что во многих важных случаях главный при $\lambda \rightarrow 0, \operatorname{Re} \lambda>0$, член разложения усредненного выражения $V(\lambda+\tilde{A})^{-1} W$ определяется первым членом разложения $(20),(21)$ (или (14)-(16)). В разобранном нами случае это дает

$$
\left\langle V \frac{1}{\widetilde{C}} \frac{1}{\lambda+\left\langle(\widetilde{C} \widetilde{B})^{-1}\right\rangle^{-1}} \widetilde{C} W\right\rangle .
$$

Для временного представления при $t \rightarrow+\infty$ отсюда следует главное приближение вида

$$
\left\langle V e^{-t \widetilde{B} \widetilde{C}} W\right\rangle \approx\left\langle V \frac{1}{\widetilde{C}} e^{-t\left\langle(\widetilde{C} \widetilde{B})^{-1}\right\rangle^{-1}} \widetilde{C} W\right\rangle .
$$




\section{4. ПРИМЕРЫ НОВЫХ МОДЕЛЕЙ}

Рассмотрим несколько примеров новых точно решаемых систем.

Начнем с описания двух операторов типа (9) с матричными элементами $U_{x-y}$ и $V_{x-y}$ вида (суммы по $k$ конечны и Т обозначает транспонирование)

$$
\left(\begin{array}{cc}
b_{x-y} & \sum_{k}\left(\beta_{x}^{1 k} f_{x-y}^{1 k}+f_{x-y}^{2 k} \beta_{y}^{2 k}+\varepsilon_{x}^{k} F_{x-y}^{k} \zeta_{y}^{k}\right) \\
g_{x-y} & h_{x-y} \gamma_{y}+\sum_{z, q} g_{x-z} b_{z-q}^{(-1)}\left[\sum_{k}\left(\beta_{x}^{1 k} f_{x-y}^{1 k}+f_{q-y}^{2 k} \beta_{y}^{2 k}+\varepsilon_{q}^{k} F_{q-y}^{k} \zeta_{y}^{k}\right)\right]
\end{array}\right)
$$

и

$$
\left(\begin{array}{cc}
p_{x-y} & \sum_{k}\left(s_{x-y}^{1 k} \eta_{y}^{1 k}+\eta_{x}^{2 k} s_{x-y}^{2 k}+\theta_{x}^{k} S_{x-y}^{k} \nu_{y}^{k}\right) \\
r_{x-y} & \mu_{x} w_{x-y}+\sum_{z, q}\left[\sum_{k}\left(s_{x-y}^{1 k} \eta_{y}^{1 k}+\eta_{x}^{2 k} s_{x-z}^{2 k}+\theta_{x}^{k} S_{x-z}^{k} \nu_{z}^{k}\right)\right] p_{z-q}^{(-1)} r_{q-y}
\end{array}\right)^{\top}
$$

При этом предполагается, что выполнены следуюшие условия:

1) заданные на решетке функции $b_{x}, b_{x}^{(-1)}, f_{x}^{1 k}, f_{x}^{2 k}, F_{x}^{k}, g_{x}, h_{x}, p_{x}, p_{x}^{(-1)}, r_{x}, s_{x}^{1 k}$, $s_{x}^{2 k}, S_{x}^{k}, w_{x}$ определяют ограниченные операторы (вида $P_{x} \rightarrow \sum_{y} a_{x-y} p_{y}$ ), причем пары $\left(b_{x}, b_{x}^{(-1)}\right)$ и $\left(p_{x}, p_{x}^{(-1)}\right)$ соответствуют обратным друг другу операторам;

2) функции $F_{x}^{k}$ и $S_{x}^{k}$ почти всюду нулевые;

3 ) соответствуюшие функциям $h_{x}$ и $w_{x}$ операторы преобразуют в себя некоторое всюду плотное подпространство пространства $l_{2}\left(\mathbb{Z}^{d}\right)$ решеточных функций, на котором они обратимы; их фурье-образы $h(k)=\sum_{x} e^{i k x} h_{x}$ и $w(k)=\sum_{x} e^{i k x} w_{x}$ при $k \neq 0$ отличны от нуля, а при $k \simeq 0$ имеют порядки $h(k)=O\left(|k|^{\Delta_{1}}\right)$ и $w(k)=O\left(|k|^{\Delta_{2}}\right)$, причем $\Delta_{1}+\Delta_{2}<d$

4) распределение заданного в каждой точке $x$ набора ограниченных случайных величин $\left(\gamma_{x}, \mu_{x} ; \beta_{x}^{1 k}, \beta_{x}^{2 k}, \varepsilon_{x}^{k}, \zeta_{x}^{k}, \eta_{x}^{1 k}, \eta_{x}^{2 k}, \theta_{x}^{k}, \nu_{x}^{k} \mid k\right)$ не зависит от точки, причем взятые в разных точках наборы независимы;

$5)$ величины $\left(\gamma_{x}\right)^{-1}$ и $\left(\mu_{x}\right)^{-1}$ имеют сколь угодно много конечных моментов.

Кроме того, должно выполняться одно из нижеследующих требований на генераторы $\tilde{A}=U V$ предлагаемых АТР-моделей.

А. Взяв неслучайный оператор $V$ (т.е. когда $\mu_{x}, \eta_{x}^{1 k}, \eta_{x}^{2 k}, \theta_{x}^{k}, \nu^{k}$ равны единице), потребуем выполнения одного из условий: $\gamma=1$ или $f^{1 k}=0$. Уже в этом случае возникают нетривиальные обобщения известных моделей случайных блужданий в неупорядоченных средах.

Б. Двойственным образом, взяв неслучайный оператор $U$, потребуем выполнения одного из условий: $\mu=1$ или $s^{1 k}=0$.

Разнообразные классы систем получаются уже при дополнительном требовании, что функции $b_{x}^{(-1)}$ или $p_{x}^{(-1)}$ почти всюду нулевые.

В. Выберем оба оператора $U$ и $V$ случайными, с ограничениями либо $s^{2 k}=0$ и $\mu=1$, либо $s^{1 k}=0$ и $s^{2 k}=0$, либо $f^{2 k}=0$ и $\gamma=1$, либо $f^{1 k}=0$ и $f^{2 k}=0$.

Для того чтобы получать в перечисленных здесь системах главный член асимптотики больших времен с помошью соотношения типа (22), необходимо вычислять величины 
$\left\langle V^{-1} U^{-1}\right\rangle^{-1}$, что удобно делать, воспользовавшись для каждого из сомножителей равенством

$$
\begin{aligned}
& \left(\begin{array}{cc}
A_{11} & A_{12} \\
A_{21} & A_{21} A_{11}{ }^{-1} A_{12}+A_{2}
\end{array}\right)^{-1}= \\
& =\left(\begin{array}{cc}
A_{11}{ }^{-1}+A_{11}^{-1} A_{12} A_{2}{ }^{-1} A_{21} A_{11}-1 & -A_{11}{ }^{-1} A_{12} A_{2}{ }^{-1} \\
-A_{2}^{-1} A_{21} A_{11}{ }^{-1} & A_{2}^{-1}
\end{array}\right) .
\end{aligned}
$$

Отметим, что для оператора $U$ величина $A_{2}=h \gamma$, а для $V$ имеем $A_{2}=\mu w$. После усреднения в $\left\langle V^{-1} U^{-1}\right\rangle^{-1}$ мы придем к $(2 \times 2)$-матрице из трансляционно-инвариантных "одномерных" операторов, обрашение которой можно осушествить с помошью преобразования Фурье.

\section{5. ПРИМЕНЕНИЕ МНОГОПАРАМЕТРИЧЕСКОГО ПРЕОБРАЗОВАНИЯ ЛАПЛАСА К ИЗУЧЕНИЮ ФЛУКТУАЦИЙ И НОВЫМ КОНСТРУКЦИЯМ АТР-МОДЕЛЕЙ}

Получение асимптотик пропагаторов в системах, генераторы которых являются суммами попарно коммутирующих генераторов АТР-моделей, или в системах 6б-6г п. 2.2, а также исследование асимптотического по времени поведения пространственных флуктуаций пропагаторов АТР-моделей проводятся с применением тех же методов, что и указанные нами выше для расчета самих пропагаторов. Дополнительно необходимо лишь применить несколько независимых преобразований Лапласа.

5.1. Для исследования систем с "разрешимыми” и попарно перестановочными генераторами $\tilde{A}^{1}, \tilde{A}^{2}, \ldots, \tilde{A}^{M}$ воспользуемся очевидным соотношением

$$
e^{-t\left(\tilde{A}^{1}+\tilde{A}^{2}+\cdots+\tilde{A}^{M}\right)}=\left\{e^{-t_{1} \tilde{A}^{1}} e^{-t_{2} \tilde{A}^{2}} \ldots e^{-t_{M} \tilde{A}^{M}}\right\}_{t_{1}=\cdots=t_{M}=t} .
$$

Это соотношение подсказывает следующий план действий. Сначала произведем над каждым сомножителем правой части преобразование Лапласа со своим параметром, т.е. перейдем к величине

$$
\prod_{m=1}^{M} \int_{0}^{\infty} d t_{m} e^{-\lambda_{m} t_{m}-t_{m} \tilde{A}^{m}}=\prod_{m=1}^{M}\left(\lambda_{m}+\tilde{A}^{m}\right) .
$$

Затем для усредненного произведения резольвент с независимыми параметрами Лапласа получим асимптотику малых значений всех параметров, совершим обратные преобразования Лапласа асимптотик по всем $\lambda_{m}$ и приравняем единому параметру времени все параметры обратных преобразований Лапласа.

Этот способ может иметь дальнейшие обобщения. Допустим, что пропагатор некоторой системы является (некоммутативным) многочленом от пропагаторов систем с почти обратимыми генераторами, как это имеет место в системах типа 6б-6г п. 2.2. В этом случае к каждому моному этого многочлена можно применить описанную процедуру, основываясь на том, что

$$
\begin{aligned}
& C_{1} e^{-t \tilde{A}^{1}} C_{2} e^{-t \tilde{A}^{2}} \ldots e^{-t \tilde{A}^{M}} C_{M+1}= \\
& \quad=\left\{C_{1} e^{-t_{1} \tilde{A}^{1}} C_{2} e^{-t_{2} \tilde{A}^{2}} \ldots e^{-t_{M} \tilde{A}^{M}} C_{M+1}\right\}_{t_{1}=\cdots=t},
\end{aligned}
$$

где $C_{1}, C_{2}, \ldots$ - достаточно обшие стохастически инвариантные операторы. 
5.2. Сформулируем условия на операторы $\tilde{A}^{1}, \tilde{A}^{2}, \ldots$ в (25), при которых получаются асимптотические разложения пропагатора в системах такого типа.

Пусть имеют место представления $\tilde{A}^{k}=\widetilde{B}^{k} \widetilde{C}^{k}$. Используем разложения

$$
\begin{aligned}
& V \frac{1}{\lambda_{1}+\tilde{A}^{1}} W_{1} \frac{1}{\lambda_{2}+\tilde{A}^{2}} W_{2} \cdots=V \frac{1}{\lambda_{1}+\widetilde{B}^{1} \widetilde{C}^{1}} W_{1} \frac{1}{\lambda_{2}+\widetilde{B}^{2} \widetilde{C}^{2}} W_{2} \cdots= \\
& =\frac{V}{\lambda_{1} \lambda_{2} \ldots}\left[1-\widetilde{B}^{1}\left(\frac{1}{\widetilde{C}^{1} \widetilde{B}^{1}}\right) \frac{1}{\lambda_{1}\left(\widetilde{C}^{1} \widetilde{B}^{1}\right)^{-1}+1} \widetilde{C}^{1}\right] \times \\
& \quad \times W_{1}\left[1-\widetilde{B}^{2}\left(\frac{1}{\widetilde{C}^{2} \widetilde{B}^{2}}\right) \frac{1}{\lambda_{2}\left(\widetilde{C}^{2} \widetilde{B}^{2}\right)^{-1}+1} \widetilde{C}^{2}\right] W_{2} \ldots
\end{aligned}
$$

Далее для каждой из величин $\left(\lambda_{k} /\left(\widetilde{C}^{k} \widetilde{B}^{k}\right)+1\right)^{-1}$ берем разложение $(15)$ с остаточным членом вида

$$
\widetilde{R}_{N+1}=\left(\lambda_{k} G_{k} \widetilde{\Psi}_{k}\right)^{N+1} \frac{1}{\lambda_{k}\left(\widetilde{C}^{k} \widetilde{B}^{k}\right)^{-1}+1} .
$$

Перейдем к формулировке условий на операторы.

Условие 1 видоизменяется за счет добавления аналогичных соотношений для операторов $W_{i}$, поскольку вместо одного оператора $\widetilde{C} \widetilde{B}$ мы получили семейство операторов $\widetilde{C}_{i} \widetilde{B}_{i}$. Далее при всех $i$ величины $\xi^{1}, \xi^{2}, \ldots$ берутся из одного их списка.

Условие 2 остается без изменений.

Условие 3 получается простым увеличением списка требований, причем в каждом из произведений все сомножители $B_{s_{1}}^{r+1} G(\lambda) F_{s_{2}}^{1},\left\langle(\widetilde{C} \widetilde{B})^{-1}\right\rangle G(\lambda) F_{s_{2}}^{1}, F_{s_{1}}^{r+1} G(\lambda) C_{s_{2}}^{1}$ и $F_{s_{1}}^{1} G(\lambda) F_{s_{2}}^{r+1}$ относятся к одному сомножителю вида $\left(\lambda_{i}+\widetilde{B}^{i} \widetilde{C}^{i}\right)^{-1} W_{i}$ из левой части (26).

Условие 4 заменяется следуюшим набором требований: для любых $1 \leqslant n_{1}<$ $n_{2}<\cdots<n_{k} \leqslant M$ величины

$$
\begin{aligned}
& \sum_{z_{1}, \ldots, z_{k} ; l_{1}, \ldots, l_{k}}\left\langle\left\{\left(\frac{\lambda_{n_{1}}}{\widetilde{C}^{n_{1}} \widetilde{B}^{n_{1}}}+1\right)^{-1} \widetilde{C}^{n_{1}} W_{n_{1}}\right\}_{z_{1} y}^{l_{1} n} \times \cdots\right. \\
& \cdots \times\left\{\left(\frac{\lambda_{n_{k}}}{\widetilde{C}^{n_{k}} \widetilde{B}^{n_{k}}}+1\right)^{-1} \widetilde{C}^{n_{k}} W_{n_{k}}\right\}_{z_{k} y}^{l_{k} n} \times \\
& \left.\times \overline{\left\{\left(\frac{\lambda_{n_{k}}}{\widetilde{C}^{n_{k}} \widetilde{B}^{n_{k}}}+1\right)^{-1} \widetilde{C}^{n_{k} W_{n_{k}}}\right\}_{z_{k} y}^{l_{k} n} \ldots\left\{\left(\frac{\lambda_{n_{1}}}{\widetilde{C}^{n_{1}} \widetilde{B}^{n_{1}}}+1\right)^{-1} \widetilde{C}^{n_{1}} W_{n_{1}}\right\}_{z_{1} y}^{l_{1} n}}\right\rangle
\end{aligned}
$$

определены при $\operatorname{Re} \lambda \geqslant 0, \lambda_{1} \lambda_{2} \cdots \neq 0$, причем при $\lambda \rightarrow 0$ и $\lambda \rightarrow \infty$ эти величины растут не быстрее некоторых степеней $\lambda$.

Еще раз отметим, что в соответствии с выбором в п. 3.3 одной из четырех возможностей мы для краткости ограничились здесь лиш ее обобшением. Соотношения (26) и (27) позволяют рассматривать сочетания различных возможностей в разных сомножителях. 
5.3. Пусть нам необходимо исследовать асимптотику пространственных флуктуаций пропагатора. Начнем со случая, когда в построении генератора не используется сложение попарно коммутирующих операторов и сам пропагатор не является суммой мономов типа (25) (обобщение на эти случаи будет достаточно очевидным).

Рассмотрим, например, асимптотику больших времен некоторой (натуральной) степени уклонения матричного элемента, т.е. величину

$$
\Delta^{2}=\left\langle\left\{\left(\widetilde{P}_{x y}^{m n}(t)-\left\langle\widetilde{P}_{x y}^{m n}(t)\right\rangle\right) \overline{\left(\widetilde{P}_{y x}^{n m}(t)-\left\langle\widetilde{P}_{y x}^{n m}(t)\right\rangle\right)}\right\}^{M}\right\rangle .
$$

После разложения правой части в сумму произведений матричных элементов экспонент генератора мы вновь получаем произведения нескольких функций от времени с определенной последовательностью усреднений в сомножителях. Естественно воспользоваться для каждого из этих сомножителей своим преобразованием Лапласа. Другими словами, мы вновь предлагаем сначала рассмотреть произведения резольвент со своими спектральными параметрами, затем произвести асимптотическое разложение соответствуюшего среднего, взять все обратные преобразования Лапласа и приравнять их параметры одному и тому же параметру $t$. Что же касается уточнений условий $1-4$ (см. п. 5.2), то здесь необходимо учесть присутствие в каждом сомножителе нескольких усреднений.

В качестве основного примера укажем, что в главном порядке асимптотика среднеквадратичного уклонения $\Delta$ матричного элемента $\widetilde{P}_{x y}^{m n}(t)$ в рассмотренных нами системах п. 3.3, где (22) дает главный член асимптотики пропагатора, и прежде всего в системах, изученных в работах [10], [14], асимптотика имеет заметно больший порядок малости. Главный порядок малости величины $\Delta^{2}$ при $t \rightarrow+\infty$ определяется выражением

$$
\begin{aligned}
& \left\langle\left[\frac{1}{\widetilde{C}} \frac{1}{\left\langle\lambda_{1}(\widetilde{C} \widetilde{B})^{-1}\right\rangle+1}\left(\left\langle\frac{1}{\widetilde{C} \widetilde{B}}\right\rangle-\frac{1}{\widetilde{C} \widetilde{B}}\right) \frac{1}{\left\langle\lambda_{1}(\widetilde{C} \widetilde{B})^{-1}\right\rangle+1}\right]_{x y}^{m n} \times\right.
\end{aligned}
$$

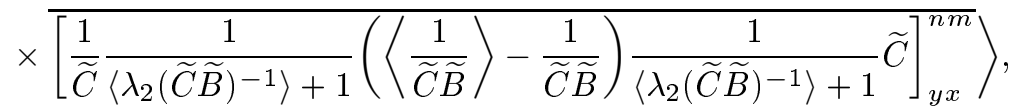

к которому следует применить обратное преобразование Лапласа по каждой из переменных $\lambda_{1}$ и $\lambda_{2}$.

5.4. В случаях задач (24) или (25) для исследования асимптотики пространственных флуктуаций следует ввести большее число параметров Лапласа. Действительно, необходимо взять по несколько параметров для каждого участвуюшего в данном мономе матричного элемента, полагая эти параметры в каждом сомножителе монома независимыми друг от друга и от параметров других сомножителей.

\section{6. ОБ ОДНОМ ЧИСЛЕННОМ МЕТОДЕ И ЕГО ПРИЛОЖЕНИИ К МОДЕЛИРОВАНИЮ НОВЫХ СИСТЕМ}

Здесь мы обсудим один метод численно-аналитического исследования случайных процессов в случайных решеточных средах [11]-[13], [15] и его применение к предложенным нами АТР-системам. 
Допустим, что среда является не вполне случайной, а периодической по каждой координате с одним и тем же большим, но конечным периодом $R$. Это означает, что случайные генераторы, будучи представлены в виде (2), удовлетворяют следующему условию: для любых векторов $x, z, w$ справедливы равенства

$$
\alpha_{x-z, x+R w} \beta_{x-z, z+R w}=\alpha_{x-z, x} \beta_{x-z, z} .
$$

Это свойство генератора имеет место по меньшей мере в том случае, если им обладают исходные и вспомогательные операторы, участвуюшие в предложенных конструкциях. Перейдем от уравнения (1) к уравнению на величины вида

$$
\rho_{x}=\sum_{y} \widetilde{P}_{x y} e^{i k(x-y)}
$$

(верхние индексы подразумеваются), в результате чего уравнение примет вид

$$
\frac{d \rho_{x}}{d t}=-\sum_{z} \tilde{A}_{x z} e^{i k(x-z)} \rho_{z},\left.\quad \rho_{x}(t)\right|_{t=0}=1 .
$$

Периодичность среды влечет соотношения $\widetilde{P}_{x+R w, y+R w}=\widetilde{P}_{x y}$ и $\rho_{x+R w}=\rho_{x}$, из которых следует конечная система уравнений

$$
\frac{d \rho_{x}}{d t}=-\sum_{z \in V}\left(\sum_{w \in \mathbb{Z}^{d}} \tilde{A}_{x, z-R w} e^{i k(x-z-R w)}\right) \rho_{z},\left.\quad \rho_{x}(t)\right|_{t=0}=1,
$$

в которой $x$ и $z$ пробегают куб периодичности $V$ - множество узлов решетки, каждая координата которых лежит между 1 и $R$.

Асимптотика решений системы (29) в ряде важных для физики приложений оказывается хорошим приближением для фурье-образа пропагатора и дает достаточную информацию об асимптотике самого пропагатора. Одним из основных доводов в пользу достоверности результатов такого моделирования является то обстоятельство, что решение выходит на асимптотическую стадию прежде, чем оно, начавшись в точке, рассматриваемой в качестве центра какого-нибудь куба периодов, существенно приблизится к его границе.

Если мы применим такой же подход к построенным в данной работе АТР-моделям, то легко убедимся в том, что на всех стадиях нам придется иметь дело с конечномерными матричными генераторами, для которых ограничение периодической среды в пределе $R \rightarrow \infty$ есть не что иное, как еще одно возмушение, которое никак не лишает их свойства почти обратимости. Другими словами, мы можем исследовать и рассчитывать процессы в периодической среде теми же методами, что и в исходной ситуации, и проверять непрерывность параметров асимптотики при $R \rightarrow \infty$.

Благодарности. Авторы признательны Р. А. Минлосу за внимание к работе и поддержку. Работа выполнена при поддержке Российского фонда фундаментальных исследований (проект № 00-01-00271-а). 


\section{Список литературы}

[1] J. Bernasconi, S. Alexander, R. Orbach. Phys. Rev. Lett. 1978. V. 41. P. 185.

[2] Я. Г. Синай. Теория вероятн. и ее примен. 1982. Т. 27. С. 247.

[3] P. J.H. Denteneer, M. H. Ernst. Phys. Rev. 1984. V. 29. P. 1755.

[4] J.-P. Bouchaud, A. George. Phys. Rep. 1990. V. 185. P. 127.

[5] Ю.Г. Абов, М.И. Булгаков, С. П. Боровлев и др. ЖЭТФ. 1991. Т. 99. С. 962.

[6] Ф. С. Джепаров. ЖЭТФ. 1991. Т. 99. С. 982.

[7] J. Bricmont, A. Kuppiainen. Phys. Rev. Lett. 1991. V. 66. P. 1689; Commun. Math. Phys. 1991. V. 142. P. 345.

[8] J. W. Haus, K. W. Kehr. Phys. Rev. B. 1991. V. 44. P. 4341.

[9] Proc. of 4th Int. Conf. on Hopping and Related Phenomena. Philos. Mag. B. 1992. V. 65. № 4.

[10] Ф. С. Джепаров, В. Е. Шестопал. ТМФ. 1993. Т. 94. С. 496.

[11] Ф. С. Джепаров, Д. В. Львов, К. Н. Нечаев, В. Е. Шестопал. Письма в ЖЭТФ. 1995. T. 62 . C. 639 .

[12] D. V. L'vov, V. E. Shestopal. Surv. High Energy Phys. 1998. V. 13. P. 223.

[13] Ф. С. Джепаров, Д. В. Львов, В. Е. ШІестопал. ЖЭТФ. 1998. Т. 114. № 6(12). С. 2166.

[14] B. E. Шестопал. ТМФ. 1999. Т. 119. С. 332.

[15] F. Dzheparov, A. Gul'ko, P. Heitjans, D. L'vov, A. Schirmer, V. Shestopal, S. Stepanov, S. Trostin. Physica B. 2001. V. 297. P. 288.

[16] М. Ф. Федорюк. Асимптотика: интегралы и ряды. М.: Наука, 1987.

Поступила в редакцию 28.XI.2001 г., после доработки 13.IX.2002 г. 